\title{
Costal cartilage resection for the treatment of slipping rib syndrome (Cyriax syndrome) in adults
}

\author{
Antonio Mazzella ${ }^{1 \#}$, Ludovic Fournel ${ }^{1 \#}$, Antonio Bobbio ${ }^{1}$, Aurélie Janet-Vendroux ${ }^{1}$, Filippo Lococo ${ }^{2}$, \\ Emelyne Canny Hamelin ${ }^{1}$, Philippe Icard ${ }^{1}$, Marco Alifano ${ }^{1}$ \\ ${ }^{1}$ Thoracic Surgery Department, Paris Center University Hospital, Assistance Publique - Hôpitaux de Paris, Paris Descartes University, Paris, France; \\ ${ }^{2}$ Department of Thoracic Surgery, Azienda Unità Sanitaria Locale - IRCCS di Reggio Emilia, Reggio Emilia, Italy \\ Contributions: (I) Conception and design: A Mazzella, L Fournel, M Alifano; (II) Administrative support: M Alifano, A Bobbio; (III) Provision of study \\ materials or patients: A Mazzella, L Fournel, A Janet-Vendroux, E Hamelin; (IV) Collection and assembly of data: A Mazzella, L Fournel, A Janet- \\ Vendroux; (V) Data analysis and interpretation: A Mazzella, L Fournel, A Janet-Vendroux, M Alifano; (VI) Manuscript writing: All authors; (VII) \\ Final approval of manuscript: All authors. \\ \#These authors contributed equally to this work. \\ Correspondence to: Dr. Antonio Mazzella. Thoracic Surgery Department, Paris Center University Hospital, 20 Rue Leblanc 75015 Paris, France. \\ Email: antonio.mazzella@aphp.fr.
}

\begin{abstract}
Background: Slipping rib syndrome is an overlooked cause of low chest or upper abdominal pain. Costal cartilage excision has been described as an effective treatment of this disorder. We review our experience with surgically treated slipping rib syndrome in the adult patient.

Methods: This is a single institution retrospective analysis from January 2000 to March 2019 of adult patients operated on for treatment of a slipping rib syndrome.

Results: Nineteen patients were diagnosed with slipping rib syndrome and underwent costal cartilage excision. All patients presented with unilateral and life disturbing chest pain (8 left sided). In all cases, point tenderness was observed with palpation and hooking maneuver was positive. Each patient underwent imaging and ultrasonography suggested slipping rib syndrome in one case. A mean of $1 \pm 0.2$ cartilages was excised. Early postoperative course was uneventful in all the cases. Follow-up was complete for all patients over a median of $18.7 \pm 12$ [3-132] months. At postoperative month 2 follow-up, 15 on 19 patients had complete resolution of their symptoms. At late interviews, 6 out of 19 patients described recurrent pain, whose intensity was significantly lower. We observed significant differences about pre-operative and postoperative visual analog pain (EVA) $(8.07 \pm 0.75$ vs. $2 \pm 2.3, \mathrm{P}<0.005)$, weekly pain crises $(6.25 \pm 2.7$ vs. 1.6 \pm 2.1 , $\mathrm{P}<0.005)$ and morphinics consomption $(9 / 19$ vs. $2 / 19, \mathrm{P}=0.029)$. Fourteen patients out of 19 nineteen strongly recommended surgical intervention.

Conclusions: Slipping rib syndrome of the adult is an overlooked cause of chest or abdominal pain which diagnosis and treatment are often delayed. Costal cartilage excision allows short to mid-terms effective and reliable treatment to reduce symptoms and life disturbance but does not exclude late pain recurrence.
\end{abstract}

Keywords: Costal cartilage; chest wall; slipping rib syndrome

Submitted Jul 17, 2019. Accepted for publication Jul 18, 2019.

doi: $10.21037 /$ jtd.2019.07.83

View this article at: http://dx.doi.org/10.21037/jtd.2019.07.83

\section{Introduction}

Slipping rib syndrome (SRS) is a mechanical cause of pain in the region of the costal cartilages, in the upper abdomen or lower chest. This painful syndrome is thought to arise from subluxation of costal cartilage tips leading to intercostal nerves impinging. The diagnosis is often difficult and underestimated because other etiologies (pleural, cardiac and gastroenterological) are considered first. 
In 1919, Cyriax described a syndrome with chest pain overlying an affected chondrocostal area that could be associated with a popping or clicking sensation (1). Later, other names have been given to this disorder as clicking or painful rib syndrome. Symptoms associated with SRS are not specific enough and lead to underdiagnosis (2). Multiple differential diagnoses encompass a clinical picture of SRS, such as costochondritis or Tietze syndrome (generally involving more cranial ribs), that is why patients might be exposed to extensive imaging evaluations or interventions before being given the right diagnosis $(3,4)$.

In SRS pain is caused when the lower cartilages (generally ribs 7 to 10 , mostly rib 8) lose their cartilaginous or fibrous attachments to each other. This condition facilitates the anterior or posterior subluxation of the ribs during movements like deep breathing, sneezing, coughing or sitting. The pain arises from the impingement of the intercostal nerve running over the adjacent rib and it is described like sharp, stabbing or burning. It can last from a few minutes to some hours $(5,6)$. We actually don't know the real prevalence of SRS, because his underdiagnosis but this condition can affect patient at any age.

Patients diagnosed with SRS are usually treated with analgesic therapy, physiotherapy or other conservative procedures but a surgical treatment might be indicated in cases of failure of the initial therapy. Indeed, DaviesColley first described in 1922 costal cartilage excision as an effective treatment of SRS (7). Since then, other authors reported cases of SRS surgically treated, but most of the time in small size and often pediatric series. In this study, we aim to describe a series of patients treated by cartilage resection reporting diagnostic tools and functional results after surgery in heterogeneous population.

\section{Methods}

A retrospective case series was compiled from a thoracic surgery department including all patients who were diagnosed with SRS and who underwent a surgical treatment from November 2000 to March 2019. Interventions consisted in cartilage excision with or without costal excision. Level and size of cartilage excision depended on pre-operative diagnostic work-up and perioperative findings. Preoperatively, when an intercostal nerve block was performed it consisted in an injection of $1 \%$ lidocaine (20 to $40 \mathrm{~mL}$ ) at the level of the offending cartilage. If there was a pain relief after block placement and reproduction of previously painful movements by the patient, the test was considered positive.

Data collected included clinical presentation characteristics, imaging evaluations, preoperative treatments, intervention type, early postoperative course and clinical results of surgery. Follow-up consisted in patients' review and examination and telephone calls. We draw up a satisfaction questionnaire; the patients were questioned about different parameters before and after surgery:

* Pain [visual analog pain (EVA) 1-10, quality of pain];

* Frequency, duration and intensity of painful crises in a week;

* Pain's impact on work (necessity of stopping work);

* Necessity of antalgic and morphinic drugs;

* Satisfactory index [1-10];

* Recommending surgery for other patients.

Continuous variables are reported as mean and standard deviation; categorical variables are reported as frequency and proportion. The statistical analysis has been performed by $\chi^{2}$ test for categorical variables and Student's $t$ test and Mann-Whitney test for continuous variables, utilizing SPSS (IBM SPSS Statistics, Version 21.0. Armonk, NY: IBM Corp. Released 2012). The difference was considered as significant for $\mathrm{P}$ values $<0.05$.

All the patients gave their informed consent to study participation. The study was performed in agreement with Helsinki Declaration and principles outlined in French laws on biomedical research.

\section{Results}

Twenty-two patients were referred for surgery to our team with diagnosis of SRS. In three of them, final diagnosis was different: one had a metastatic fracture of the right ninth rib from a primary thyroid cancer; another had a lower chest-upper abdominal pain finally secondary to Crohn's disease and a third one had a non-union fracture secondary to previous thoracic surgery. In nineteen patients, final diagnosis was SRS and all had costal cartilage excision with intraoperative findings consistent with the disease. There were eight men and eleven women with a median age at diagnosis of $41 \pm 17$ [19-82] years. The median time from the onset of symptoms to surgery was 2.5 [1-10] years. Three patients reported a history of former trauma with hospitalization but without direct chest wall injury. All patients presented with unilateral lower chest pain which was life disturbing (11 right and 8 left-sided). In sixteen cases, SRS was associated with sleep difficulties or 
Table 1 Clinical and demographic characteristics

\begin{tabular}{llllllcc}
\hline Patient & Age & Sex & Level of point tenderness & Side & $\begin{array}{c}\text { Pain with } \\
\text { activity? }\end{array}$ & $\begin{array}{c}\text { Popping/clicking } \\
\text { sensation? }\end{array}$ & Subluxation? \\
\hline 1 & 43 & W & T8 & Right & Yes & Yes & Yes \\
2 & 82 & W & T9 & Left & Yes & No & Yes \\
3 & 25 & M & T9 & Right & Yes & No & Yes \\
4 & 52 & M & T8 & Left & Yes & Yes & Yes \\
5 & 36 & M & T10 & Right & Yes & No & Yes \\
6 & 58 & W & T10 & Right & Yes & Yes & Yes \\
7 & 64 & W & T8 & Right & Yes & Yes & Yes \\
8 & 28 & M & T11 & Left & Yes & Yes & Yes \\
9 & 23 & W & T9 & Right & Yes & Yes & Yes \\
10 & 23 & M & T9 & Right & Yes & Yes & Yes \\
11 & 26 & M & T11 & Right & No & Yes & Yes \\
12 & 28 & W & T10 & Left & No & Yes & No \\
13 & 42 & M & T7-8 & Left & Yes & Yes & Yes \\
14 & 61 & W & T10 & Right & No & Yes & Yes \\
15 & 19 & W & T8 & Left & Yes & Yes & Yes \\
16 & 37 & W & T8 & Right & Yes & No & No \\
17 & 36 & W & T10 & Right & Yes & No & Yes \\
18 & 39 & M & T8 & Right & Yes & No & Yes \\
\hline 19 & 58 & W & T10 & Right & Yes & Yes & Yes \\
\hline
\end{tabular}

disturbances. Nine patients described troubles in work or study course that they directly attributed to their disorder. At preoperative physical examination, point tenderness was always observed and the hooking maneuver was positive in all cases. Clinical signs and symptoms are summarized in Table 1 .

Each patient was referred to our institution with a full preoperative diagnostic work-up. Computed tomography scan was performed in all cases and didn't reveal any costal cartilage or chest wall abnormality. Nine patients underwent a $(99 \mathrm{~m})$ Tc MDP scintigraphy which was always normal and didn't show any significant uptake. A thoracic and lumbar magnetic resonance imaging was also performed in six cases and excluded any spine, sternal or chest wall disorder. Among five patients who underwent an ultrasonography, only two had an abnormal imaging which demonstrated a dynamic subluxation of the rib, suggesting a SRS. In one patient with right upper abdominal pain, a diagnostic laparoscopy was performed and resulted normal. All patients had been previously referred to other specialists who initiated conservative medical procedures. Oral analgesic therapy had been attempted in all the cases, including morphine-derivate drugs, tramadol, non-steroidal anti-inflammatory or benzodiazepine. All the patients referred to our department presented with pain recurrence or persistence despite medical treatment. Sixteen intercostal blocks had been performed before surgery and had led to transient pain relief.

With respect to surgical procedure, a mean of $1 \pm 0.2$ cartilages were resected. The costal cartilage that corresponded to point tenderness and that was included in excision was located at level T8 in six patients, T9 in four patients, T10 in six patients, T11 in two patient and T7 and T8 in one patient (Figures 1,2). Costal cartilage excision was associated with short rib resection in 15 cases. Median size of costal cartilage and rib resection was 3.6 $\pm 1.1(1.8-7.5)$. All removed cartilages presented with an abnormality such as mobility and luxation. On pathologic review, macroscopic 

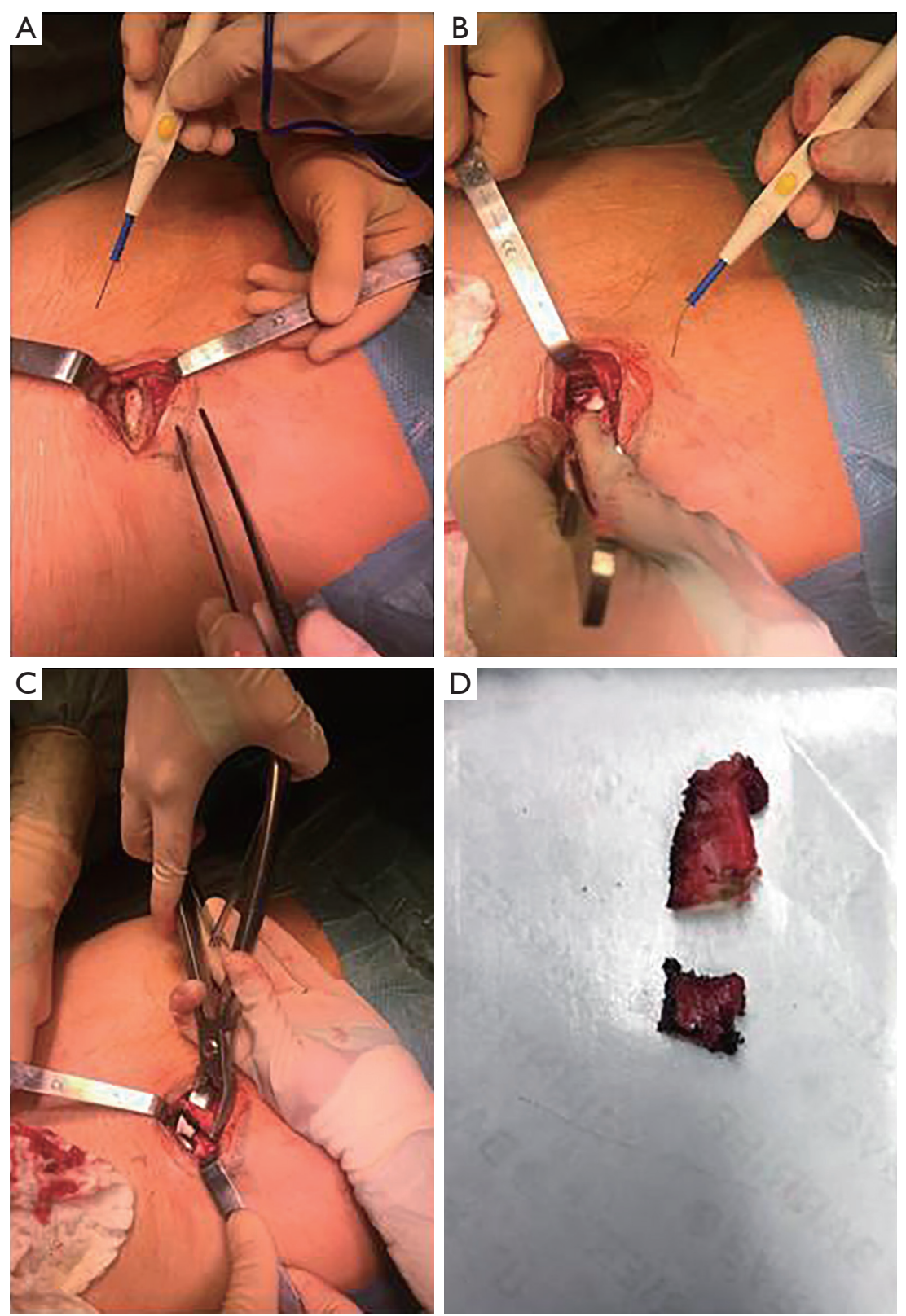

Figure 1 Operative phases of resection. (A,B) Isolation of perichondrium, costal cartilage and rib; (C) resection of costal cartilage and adjacent rib; (D) intraoperative specimen (costal cartilage and rib).

appearance of cartilages was always normal and histologic analysis revealed normal cartilaginous or bone tissue. The early postoperative course was uneventful and median hospital stay was 3 [2-4] days.

Follow-up was complete for all patients over a median of $18.7 \pm 12$ [3-132] months. At postoperative month 2 follow-up, 15 on 19 patients had complete resolution of their previous presenting symptoms. These early interviews revealed complete pain relief and vanishing of luxation, popping or any other SRS related sensation. At late interviews, 6 on 19 patients described recurrent pain in the same location but much less severe and less life disturbing. These pain relapses didn't occur at rest but only during movement or palpation. In one computer scientist, operation didn't allow return to work because of a lingering pain at sitting position. 
We observed significant differences about preoperative and late post-operative EVA $(8.07 \pm 0.75$ vs. $2 \pm 2.3$, $\mathrm{P}<0.005)$, weekly pain crises $(6.25 \pm 2.7$ vs. $1.6 \pm 2.1, \mathrm{P}<0.005)$ and morphine consomption $(9 / 19$ vs. $2 / 19, \mathrm{P}=0.029)$ (Table 2). Fourteen patients on nineteen strongly recommended surgical intervention.

\section{Discussion}

SRS is an overlooked cause of chest or abdominal pain. Difficulty in diagnosing this disorder is mainly related to non-specific symptoms or imaging and to an unclear etiology. In most instances SRS is attributed to a hypermobile rib, secondary to intercostal articulation weakness, which slips under an adjacent rib leading to focal and severe pain (9). The loosened rib may sublux with certain movements, activities or with influences of physical examination. Reproduction of the pain event thanks to an induced rib subluxation is a key clinical testing (10). All patients from our series had a positive "hooking maneuver" which reveals to be very useful to confirm clinical suspicion.

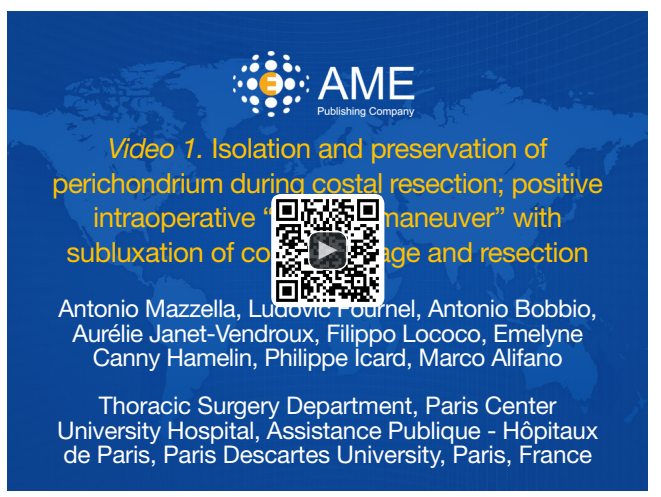

Figure 2 Isolation and preservation of perichondrium during costal resection; positive intraoperative "hooking maneuver" with subluxation of costal cartilage and resection (8).

Available online: http://www.asvide.com/watch/33046
Unlike other similar syndromes, SRS is mainly considered as a primary non-inflammatory process (3). In our series, absence of chronic or acute inflammatory signs on pathologic findings from the resected cartilage may support this observation. Unfortunately, the histological examination of resected costal cartilages is poorly reported and distinction between SRS and inflammatory disorders of the costal cartilage such as costochondritis could not be well appreciated in the current literature. As pathogenesis and diagnosis of this clinical entity remain unclear, some cases of pure SRS were most probably reported as costochondritis and vice versa. This may explain contradictory results about diagnosis and treatment of this syndrome.

Few series, including a small number of patients, describe the clinical expression of SRS. Barki et al. reported the largest retrospective study, including 100 cases of painful rib syndrome (11). This descriptive analysis led to the conclusion that diagnosis of SRS is based on the presence of pain upon applying pressure to the anterior and inferior border of the rib cage and that this disorder is often secondary to a traumatic event. The most common clinical presentation is a unilateral focal pain often associated with a popping or clicking sensation. Indeed, the rib end may sublux across the medial attachment site creating a snapping or "giving way" sensation simultaneously to a sudden exacerbation in pain (12). In our experience, a history of chronic costal cartilage pain, exacerbated by movement, isn't sufficient to make the diagnosis of SRS. Instead, what seems more convincing is a positive intercostal block test. In presence of relief with intercostal block combined with a typical clinical examination of SRS we may avoid other complementary diagnostic interventions.

In this frequently overlooked disorder, several imaging modalities have been described and tested as diagnostic tools. When patients with SRS are proposed for surgical resection, they commonly have an eventful previous diagnostic course. Thoraco-abdominal tomography and MRI are often performed to avoid differential diagnosis

Table 2 Satisfaction questionnaire

\begin{tabular}{lccc}
\hline Investigated outcomes & Pre-operative & Post-operative & P \\
\hline Visual analog pain (EVA) [1-10] & $8.07 \pm 0.75$ & $2 \pm 2.3$ & $<0.005$ \\
Weekly pain crises & $6.25 \pm 2.7$ & $1.6 \pm 2.1$ & $<0.005$ \\
Morphine consomption & $9 / 19(47 \%)$ & $2 / 19(11 \%)$ & 0.029 \\
Surgery satisfaction & - & $14 / 19(74 \%)$ & - \\
\hline
\end{tabular}


like inflammatory chest wall or abdominal disorders. In our series, only one radiologist evoked a possible SRS by performing an ultrasonography over the point tenderness. This diagnostic modality has already been described in 3 adults allowing for visualization of the subluxating cartilage (13). Positive diagnosis of SRS using ultrasonography is probably easier compared with other imaging modalities because it is dynamic and patient may reproduce the painful movement during examination. Nevertheless, it requires experienced operators and is maybe not much superior to a well conducted physical examination.

Regarding our surgical approach, we always removed the cartilage considered responsible for maximal pain. This procedure requires a precise preoperative physical examination in order to spot the cartilage to be resected. As described in other series of operated SRS, we tried to preserve the perichondrium in order to accelerate cicatrization (12). When we intraoperatively observed any other abnormal cartilage or costal segment, we extended the costal cartilage excision to more than one level. Indeed, even though resection of the affected cartilage is effective, SRS presentation isn't always classic and intraoperative observations are sometimes unclear, thus we may recommend carrying an extended resection of all abnormal cartilages from the sternum to the anterior costal extremity. The mechanical nature of this disorder was confirmed by pathologic findings that revealed normal cartilaginous or bone tissue.

Surgical costal cartilage excision led to the resolution of symptoms in the early postoperative course of all patients from this case series. This analysis corroborates results from other series of surgically treated SRS which showed an early efficacy on pain control, symptoms resolution and quality of life $(14,15)$. However, mid and long terms followup revealed late symptoms recurrence in 6 patients out of 19. Indeed, the most frequent relapsing symptom was an ipsilateral focal pain despite excision of the subluxating cartilage. Late recurrences suggest the existence of a previous non mechanical etiology partially responsible for early and late pain. When surgical excision doesn't allow late pain relief, it is very likely that the costal cartilage subluxation lead to the installation of chronic pain with neuropathic and non-organic components which can't be treated with surgery alone. We can also suppose that surgery could have induced in itself a degree of neuropathic pain which was initially relieved by increased levels of analgesic but returned after down-titration.
Nevertheless, fourteen patients on nineteen strongly recommended the surgery and they have been satisfied for the intervention, despite some late recurrences. Indeed, we have observed a considerable improvement concerning EVA, weekly pain crises and morphine-derivate consummation after surgery in these patients.

In conclusion, costal cartilage excision allows short to mid-terms effective and reliable therapy to reduce symptoms and life disturbance but don't exclude late pain recurrence. Nevertheless, this is a retrospective case series with a small number of patients which is subject to many biases. Another main limitation of our study is that our database query only identified patients surgically treated. Thus, it was impossible to compare or analyze the majority of SRS that weren't referred in our institution to be operated on. Further studies may be necessary to identify larger samples of patients with SRS in particular those with non-classic presentation or those treated with nonsurgical procedures such as neuropathic pain agents like amitriptyline or gabapentin. For the same purpose, it would be helpful to improve information about this rare and overlooked disorder.

\section{Acknowledgments}

None.

\section{Footnote}

Conflicts of Interest: The authors have no conflicts of interest to declare.

Ethical Statement: The authors are accountable for all aspects of the work in ensuring that questions related to the accuracy or integrity of any part of the work are appropriately investigated and resolved. All the patients gave their informed consent to study participation. The study was performed in agreement with Helsinki Declaration and principles outlined in French laws on biomedical research. No more ethical approval is required.

\section{References}

1. Cyriax EF. On various conditions that may simulate the referred pains of visceral disease, and a consideration of these from the point of view of cause and effect. Practitioner 1919;102:314-22.

2. Wright JT. Slipping-rib syndrome. Lancet 
1980;2:632-634.

3. Gregory PL, Biswas AC, Batt ME. Musculoskeletal problems of the chest wall in athletes. Sports Med 2002;32:235-50.

4. Lum-Hee N, Abdulla A. Slipping rib syndrome: an overlooked cause of chest an abdominal pain. Int J Clin Pract 1997;51:252-3.

5. McMahon LE. Slipping Rib Syndrome: A review of evaluation, diagnosis and treatment. Semin Pediatr Surg 2018;27:183-8.

6. Fares MY, Dimassi Z, Baydoun H, et al. Slipping Rib Syndrome: Solving the Mystery of the Shooting Pain. Am J Med Sci 2019;357:168-73

7. Davies-Colley R. Slipping rib. Br Med J 1922;1:432.

8. Mazzella A, Fournel L, Bobbio A, et al. Isolation and preservation of perichondrium during costal resection; positive intraoperative "hooking maneuver" with subluxation of costal cartilage and resection. Asvide 2020;7:002 .

Available online: http://www.asvide.com/watch/33046

Cite this article as: Mazzella A, Fournel L, Bobbio A, JanetVendroux A, Lococo F, Hamelin EC, Icard P, Alifano M. Costal cartilage resection for the treatment of slipping rib syndrome (Cyriax syndrome) in adults. J Thorac Dis 2020;12(1):10-16. doi: $10.21037 /$ jtd.2019.07.83
9. Spence EK, Rosato EF. The slipping rib syndrome. Arch Surg 1983;118:1330-2.

10. Heinz GJ, Zavala DC. Slipping rib syndrome. JAMA 1977;237:794-5.

11. Barki J, Blanc P, Michel J, et al. Painful rib syndrome (or Cyriax syndrome): study of 100 patients. Presse Med 1996;25:973-6.

12. Holmes JF. Slipping rib cartilage. Am J Surg 1941;54:326-38.

13. Meuwly JY, Wicky S, Schnyder P, et al. Slipping rib syndrome: a place for sonography in the diagnosis of a frequently overlooked cause of abdominal or low thoracic pain. J Ultrasound Med 2002;21:339-43.

14. Copeland GP, Machin DG, Shennan JM. Surgical treatment of the "slipping rib syndrome". Br J Surg 1984;71:522-3.

15. Saltzman DA, Schmitz ML, Smith SD, et al. The slipping rib syndrome in children. Paediatr Anaesth 2001;11:740-3. 